\title{
Design and Implementation Fast Correction Phase System of Ship-borne Dynamic Platform
}

\author{
Li Ren-long, WU Guang-zhi, Kou Ming-xin \\ China Satellite Maritime Tracking and Control Department, Jiangyin, 214431, China
}

Keywords: Fast Correction Phase, Ship-borne, static, dynamic

\begin{abstract}
The traditional calibration phase just can meet the he static tower condition at pier, but can not use for the dynamic target. The Fast Correction Phase model is built for Ship-borne Dynamic Platform of tracking and control radar. The anti-cosine method is put forward to calculate phase difference. Then the Fast Correction Phase System of Ship-borne Dynamic Platform is advanced. It can not only to meet the static tower condition at pier, but also to meet the dynamic way on sea. The technology can meet the dynamic target for calibration. It has many advantages, such as fast checking, adaptable for device, good performance, and universal for Land-based and ship-borne, and simple operation, easy to automate, etc. It has great value.
\end{abstract}

\section{Introduction}

Shipborne Unified Control System is the core of the battle space tracking ship monitoring and control equipment. For many of the shipboard unified control system, its tracking system are dual-channel amplitude comparison monopulse system, the tracking system due to its high accuracy, good dynamic performance, relatively low link cost and other advantages. However, dual-channel tracking system also has a significant drawback, and that is the phase difference between the road and the road between the two channels is inconsistent shift amount, can cause cross-coupling angle information demodulation, so that the self-tracking antenna automatic control loop convergence worse, serious and even lead to runaway unable to track the target.

\section{Traditional Methods and Limitations of the school phase}

Aditional methods can be divided into two phases school, marina and sea phase calibration phase calibration. The ship at the pier calibration using the tower, synchronous satellite, microwave self, marina and other partial feed derived calibration mode phase and frequency slope values corresponding point and link combinations, and recorded as a reference phase comparison, in maritime school conducted by the same phase calibration method, and the phase correction value according to the pier. The traditional method of phase correction to some extent to meet the needs of the mission, also played an important role, but security conditions and more traditional methods, school phase process is complicated, and requires sufficient time to ensure that various methods have their limitations sex.

(1). Calibration tower on school phase restricted by many factors, only for tracking ship docked at the pier and calibration, as the tower is visible through the case, the calibration tower needed personnel synergies completed school with a long time, low efficiency .

(2). Synchronous satellite carried a single frequency correction phase, in most cases with the desired frequency synchronous satellite school with a different frequency, the method can only test phase stability and relative amount of change.

(3). Using partial feed phase correction phase value obtained by the method can be used as a reference amount only when the deviation of the two calibration results, the amount of change in the value obtained for phase correction initially bound, unstable and calibration results, the method influenced by the environment.

(4). When using synchronous satellite tracking beacons and calibration ball on the sea state and weather conditions demanding, if the hull will result in a larger rock calibration deviation is larger, 
demanding staff positions.

(5). Did not fully test the performance of the tracking channel when the self-test mode microwave phase correction, in particular can not examine the effect of the feed network and the phase slope.

Traditional school phase process for standard TT \& C spectrum mode 1, 2 have a better spreading pattern phase deviation correction model, by observing the phase linearity, phase correction can be performed on the deviation, but still no less FM mode good phase deviation correction model, when the phase linearity error is large only to re-school phase column, if at sea, can only be used for qualitative way satellite link to determine whether the phase changes due to other factors such rocking boat poor accuracy of satellite correction phase, phase correction accuracy tends to be low, and the phase correction mode FM sea except put the ball outside the school with almost no viable means. Similarly, the traditional way of conducting phase correction phase linearity and sensitivity verification requires synergy between multiple positions, servo staff positions at the observation voltage linearity and sensitivity factors artificially increased subjective judgment, to bring the phase correction Uncertainties.

\section{Rapid correction phase techniques and error correction}

\section{New calibration technology based on difference}

Existing calibration methods, new rapid calibration method calibration process does not require radar antenna axis remains partial opening goal beacon a fixed angle, instead of using the difference method for angle and angular error in two different moments data calculation, obtained calibration results. Specific works as follows.

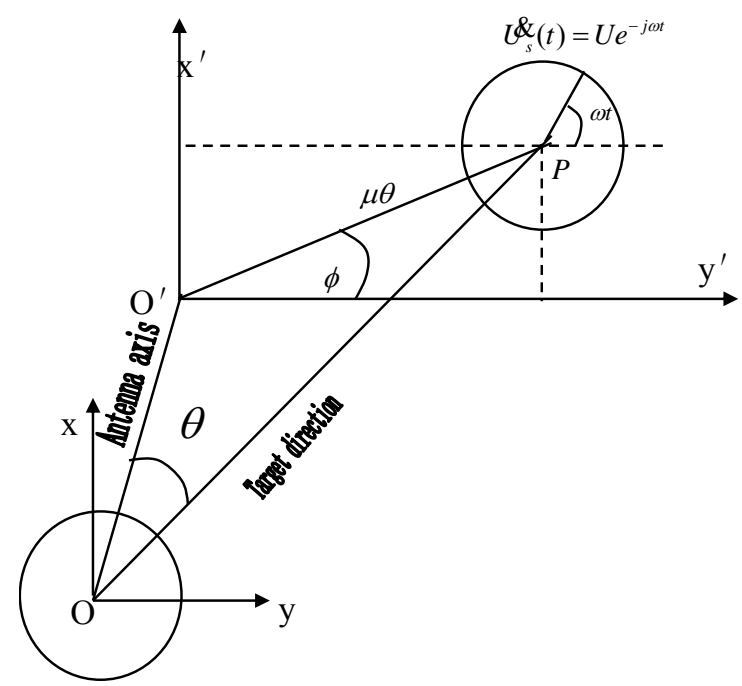

Figure 1. Forming principle of differential-mode dual-channel monopulse angle error signal

As shown, in different locations at different times radar antenna axis relative target coordinates available $(\theta, \varphi)$ represents. Two different moments of the moving target relative angle of the antenna:

$$
\left\{\begin{array}{l}
\Delta A=\theta_{1} \cdot \cos \Phi_{1}-\theta_{2} \cdot \cos \Phi_{2}=A_{1}-A_{2} \\
\Delta E=\theta_{1} \cdot \sin \Phi_{1}-\theta_{2} \cdot \sin \Phi_{2}=E_{1}-E_{2}
\end{array}\right.
$$

Consists of a series can be derived:

$$
\begin{gathered}
\Delta \phi=-\arccos \frac{x}{Z} \\
k=\sqrt{\frac{\Delta U_{E}^{2}+\Delta U_{A}^{2}}{\Delta E^{2}+\Delta A^{2}}}
\end{gathered}
$$

Tracking receiver is loaded with an arbitrary phase slope, keeping the target within the main beam range. Get target relative angle of the antenna change values. The retardation value and slope into tracking receiver, automatic calibration phase is complete. 


\section{Theoretical basis for automatic calibration with software}

The signal received by the receiver tracking form, can be divided into $\mathrm{L}$ received signal, the received signal is right-handed, FM mode while receiving signals about spin. Based on the previous section, the derivation process, the following will introduce the theoretical basis automatic calibration software, respectively, for the two-phase signal form.

a. L signal

Obtain phase tracking receiver and slope are binding initial value. Phase correction amount obtained through automatic calibration and slope coefficient with the software. Calculated true phase and slope:

$$
\begin{aligned}
& \Phi A_{L}=\Phi A_{L 0}+\Delta \Phi \\
& \Phi E_{L}=\Phi E_{L 0}+\Delta \Phi \\
& k=0.5 * k_{0} / k_{1}
\end{aligned}
$$

The new phase slope and implanted a tracking receiver, automatic calibration phase is complete.

b. Right-handed signal

Obtain phase tracking receiver and slope are binding initial value. Phase correction amount obtained through automatic calibration and slope coefficient with the software. Calculated true phase and slope:

$$
\begin{aligned}
& \Phi A_{R}=\Phi A_{R 0}-\Delta \Phi \\
& \Phi E_{R}=\Phi E_{R 0}-\Delta \Phi \\
& k=0.5 * k_{0} / k_{1}
\end{aligned}
$$

\begin{tabular}{|c|c|}
\hline $\begin{array}{c}\text { Deviation (actual value - } \\
\text { binding value) }\end{array}$ & Time \\
\hline$-30^{\circ}$ & $08: 22: 45$ \\
\hline $35^{\circ}$ & 08: $22: 50$ \\
\hline $80^{\circ}$ & 08: 23: 01 \\
\hline $135^{\circ}$ & 08: 23: 08 \\
\hline $180^{\circ}$ & $08: 23: 16$ \\
\hline$-160^{\circ}$ & 08: 23: 31 \\
\hline$-140^{\circ}$ & 08: $23: 41$ \\
\hline$-90^{\circ}$ & 08: $23: 51$ \\
\hline \multicolumn{2}{|l|}{ data source $\triangle \mathrm{Phase}\left({ }^{\circ}\right)$} \\
\hline & \\
\hline$-1-t-t$ & . \\
\hline mound _. & 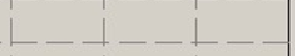 \\
\hline$(201) \quad 1$ & $\frac{1}{1}--$ \\
\hline & \\
\hline & samen \\
\hline & 1 Amandons \\
\hline $\begin{array}{l}\mid \\
\end{array}$ & Anewhent 1116 \\
\hline
\end{tabular}

The new phase slope and implanted a tracking receiver, automatic calibration phase is complete.

\section{Test Data Analysis}

Using fast phase correction algorithm proposed various conditions and with the ball to put the star test. The following part of the test results of a representative selection is as follows:

Table 1. Teeing data (tracking receiver initial phase, $\mathrm{L}$ )

Figure 2. After the pull side put the ball at different angles relative to the rapid correction software calibration results in Fig. 
Wherein a satellite tracking process, USB tracking receiver using different initial phase, as follows:

Table 2. A satellite tracking (tracking receiver initial phase)

\begin{tabular}{|c|c|c|}
\hline The initial phase & Time & Remark \\
\hline $\mathrm{L} 180^{\circ}$ & $21: 34: 15$ & \multirow{4}{*}{ Single rotation } \\
\hline $\mathrm{L} 210^{\circ}$ & $21: 34: 23$ & \\
\hline $\mathrm{R} 223^{\circ}$ & $21: 34: 30$ & \\
\hline $\mathrm{R} 83^{\circ}$ & $21: 34: 34$ & \\
\hline $\mathrm{L} 180^{\circ}$ phase $97^{\circ}$ & 21: $34: 41$ & Synthesis grading \\
\hline \multicolumn{3}{|c|}{$\begin{array}{l}\text { Note: for the correct phase: L } 180^{\circ} \text { phase difference } 97^{\circ} \text { (ie, L } \\
\qquad 180^{\circ} \text { clockwise } 83^{\circ} \text { ) }\end{array}$} \\
\hline
\end{tabular}

data source $\triangle \operatorname{Phase}\left({ }^{\circ}\right)$

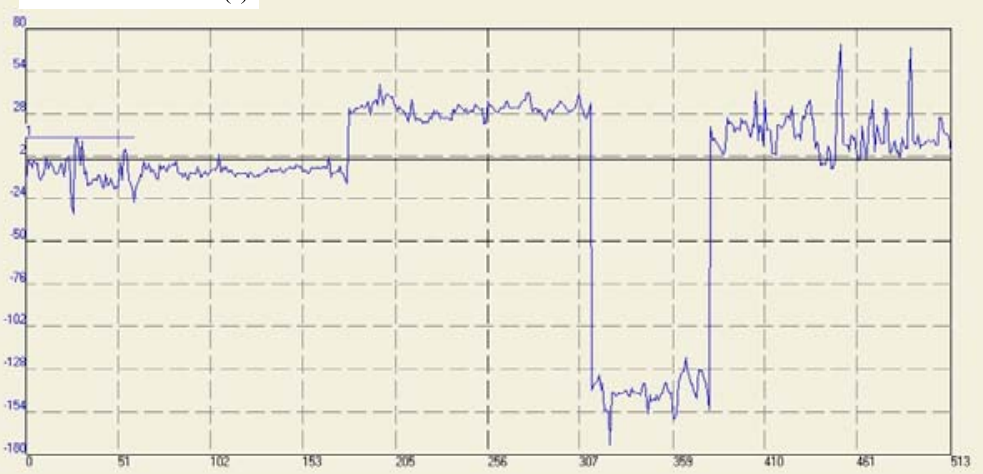

Figure 3. The satellite tracking a different binding initial phase after rapid correction phase software calibration results

Can be obtained from Fig. 2 and 3, the proposed rapid correction phase method, to meet different system, different polarization under static and dynamic conditions, phase calibration, the calibration results meets the accuracy requirements.

\section{Summary}

This paper established a rapid correction phase model shipboard monitoring and control radar moving platform, and the tracking ship under dynamic conditions during dynamic target tracking phase calibration, which not only meets the pier for a static column mode, while adapting dynamically the sea with stars school phase. Both to meet the USB and UCB fast phase calibration system, the system can meet UXB fast phase calibration. The technology has traceable calibration target dynamic, fast, without any auxiliary equipment, no advance preparation phase, the device adaptability, performance, general-purpose shipborne land-based, simple, easy to realize the advantages of automation, with a very great value.

\section{Reference}

[1] Fields T W, Sharpin D L, Tsui J B. Digital channelized IFM receiver[A]. Microwave Symposium Digest[C]. IEEE MTT—S International, vo1. 3. 2010

[2] Tsui, J.B.Y, Stephens J.P, Sr. Digital Microwave Receiver Technology[J]. IEEE Trans. -MTT, 2012 .

[3] DIETMAR M. The Digital Receiver - A Technology for Future Electronic Warfare Systems [R]. International Conference on Radar System'2008 France, May, 2008.

[4] M. Cummings and S. Haruyama: FPGA inthe Software Radio, IEEE Communications Magazine, vo1. 37, Feb. 2009 